\title{
Analysis and Performance Evaluation of an All-Fiber Wide Range Interrogation System for a Bragg Grating Sensor Array
}

\author{
Ginu Rajan \\ Technological University Dublin, ginu.rajan@tudublin.ie \\ Yuliya Semenova \\ Technological University Dublin, yuliya.semenova@tudublin.ie \\ Gerald Farrell \\ Technological University Dublin, gerald.farrell@tudublin.ie
}

Follow this and additional works at: https://arrow.tudublin.ie/engscheceart

Part of the Electrical and Computer Engineering Commons

\section{Recommended Citation}

Rajan, G., Semenova, Y. \& Farrell, G.(2009) Analysis and Performance Evaluation of an All-Fiber Wide Range Interrogation System for a Bragg Grating Sensor Array. Journal of Optics A:Pure and Applied Optics, Vol.11. doi:10.1088/1464-4258/11/5/054004

This Article is brought to you for free and open access by the School of Electrical and Electronic Engineering at ARROW@TU Dublin. It has been accepted for inclusion in Articles by an authorized administrator of ARROW@TU Dublin. For more information, please contact arrow.admin@tudublin.ie, aisling.coyne@tudublin.ie, gerard.connolly@tudublin.ie.

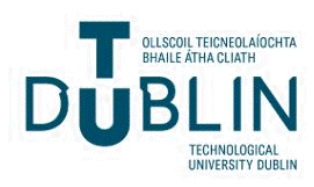




\title{
Analysis and performance evaluation of an all-fiber wide range interrogation system for a Bragg grating sensor array
}

\author{
Ginu Rajan, Yuliya Semenova, Gerald Farrell \\ Applied Optoelectronics Centre, School of Electronic and Communications \\ Engineering, Dublin Institute of Technology, Dublin 8, Ireland \\ E-mail: ginu.rajan@dit.ie
}

\begin{abstract}
Analysis and performance evaluation of a macro-bend fiber based interrogation system for a Bragg grating sensor array is presented. Due to the characteristic properties of the macro-bend fiber filter such as polarization and temperature dependence and the total noise associated with the ratiometric system, a best fit ratio slope is required to interrogate multiple FBGs whose peak wavelengths are spread over a wide wavelength range, rather than the optimal slope for individual FBG. In this paper for investigation we have used an FBG array with 5 FBGs with peak reflected wavelengths lying between $1525 \mathrm{~nm}$ and $1575 \mathrm{~nm}$. The analysis of the system is carried out and a fiber filter with a slope which covers a wavelength range of $1525-1575 \mathrm{~nm}$ is selected which ensures a resolution and accuracy for all the FBG sensors in the array as close as possible to that which would be achieved with a filter with an optimal slope for each FBG. Performance evaluation of the system is carried out and static strain, dynamic strain and temperature is measured with the developed interrogation system.
\end{abstract}

PACS numbers: $42.79,42.81$

Keywords: macro-bend fiber filter, FBG sensor, static strain, dynamic strain, temperature.

Submitted to: J. Opt. A: Pure Appl. Opt. 


\section{Introduction}

Fiber Bragg grating (FBG) sensors have generated much interest in the area of strain and temperature sensing and are being used in many applications such as structural monitoring and smart structures [1-5]. The critical aspect related to the practical use of an FBG sensor is the necessity of performing accurate measurement of the small wavelength shift associated with thermal and strain state changes. To facilitate the broad use of this class of sensor compact, rugged, wide range and low cost interrogation systems are required. In addition, improvements in the fabrication of FBGs using phase mask techniques [6] have reduced the cost of grating fabrication, and the interrogation unit, rather than the sensor accounts for a large proportion of the cost of a complete sensing system. Many techniques have been developed to interrogate FBGs based on different principles [7-11]. An interrogation system based on a macro-bending singlemode fiber edge filter [12] offers the advantages of an all-fiber approach with low cost together with large dynamic range and wide wavelength measurement range. Due to the wide wavelength range such a system can interrogate multiple FBGs when used together with a fast optical tunable filter.

Earlier investigations of the properties of a macro-bend fiber filter showed that a macro-bend fiber exhibits polarization dependent loss (PDL) $[13,14]$ and temperature dependent loss (TDL) $[15,16]$ and both these phenomena are functions of wavelength and also depend on the slope of the system which is determined by the bend radius and number of bend turns of the fiber filter. Furthermore the signal-to-noise ratio (SNR) of the source and the noise in the receiver limits the resolution of the system [17]. It is proved that the noise induced ratio fluctuation is wavelength dependent and hence the resolution of the system itself is wavelength dependent. Overall the accuracy and resolution of the interrogation system will be different for different wavelengths and will be dependent on the edge filter slope. To interrogate a single FBG the slope of the system can be optimized so it has the highest resolution and accuracy. However for an array of FBG sensors with peak wavelengths spread over a wide range it is not possible to adopt a single slope that yields the best resolution and accuracy for all the FBGs. In this paper we present an analysis that takes account of all the factors affecting the resolution and accuracy of the system in order to select a fiber filter with a best fit slope which gives the highest possible resolution and accuracy for an array of FBG sensors. A brief description of the basic principles of FBG sensing is presented in section 2 . An interrogation system based on macro-bend fiber filter and its analysis and criteria for selection of a suitable fiber filter for an FBG array are presented in section 3. A performance evaluation of the system has been carried out and is presented in section 4. 


\section{Principles of FBG sensing}

The operation of an FBG sensor is based on the measurement of the peak wavelength shift induced by the applied strain or change in temperature [18]. The light reflected by periodic variations of the refractive index of the Bragg grating having a central wavelength $\lambda_{G}$ is given by $\lambda_{G}=2 \mathrm{n} \Lambda$, where $\mathrm{n}$ is the effective refractive index of the core and $\Lambda$ is the periodicity of the refractive index modulation. The sensitivity of the Bragg wavelength to temperature arises from the change in period associated with the thermal expansion of the fiber, coupled with a change in the refractive index arising from the thermo-optic effect. The strain sensitivity of the Bragg wavelength arises from the change in period of the fiber grating coupled with a change in the refractive index arising from the strain-optic effect.

For the measurement of a temperature change $\Delta \mathrm{T}$, the corresponding wavelength shift is given by $\Delta \lambda_{T}=\lambda_{G}(\alpha+\xi) \Delta T$, where $\alpha$ is the coefficient of thermal expansion of the fiber material and $\xi$ is the fiber thermo optic coefficient.

For the measurement of applied longitudinal strain, the wavelength shift is given by $\Delta \lambda_{S}=\lambda_{G}\left(1-\rho_{\alpha}\right) \Delta \varepsilon$, where $\rho_{\alpha}$ is the photo elastic coefficient of the fiber given by the formula $\rho_{\alpha}=\frac{n^{2}}{2}\left[\rho_{12}-v\left(\rho_{11}-\rho_{12}\right)\right]$, where $\rho_{11}$ and $\rho_{12}$ are the components of fiber optic strain tensor and $v$ is the Poisson's ratio. For a silica core fiber the value of (1 $-\rho_{\alpha}$ ) is usually 0.78 . Thus by measuring the wavelength shift changes in temperature or strain can be determined.

\section{An interrogation system based on a macro-bend fiber filter}

The bend loss wavelength characteristic of a macro bend fiber allows it to be employed as the core of an interrogation system for FBG sensors when used in a ratiometric scheme [12]. A schematic of the experimental interrogation system used in this investigation for an array of FBG sensors based on macro-bend fiber filter is shown in Fig. 1. A super luminescent diode was used as a broadband source (BBS) to drive the Bragg gratings. An optical isolator is used to block any reflected light from reaching the source. An optical circulator is used to direct the reflected signal from the FBGs to the demodulation system. Any edge filter based ratiometric demodulation system can measure only one wavelength at a time. Therefore an optical tunable wavelength filter is used to filter the reflected wavelengths, extracting a single wavelength at a time which is then inputed into the demodulation system, whose band pass allows it to measure the wavelength shift induced by strain or temperature variations.

Five FBGs with peak reflected wavelengths at $1530.40 \mathrm{~nm}, 1540.15 \mathrm{~nm}, 1550.2 \mathrm{~nm}$, $1560.1 \mathrm{~nm}, 1571.4 \mathrm{~nm}$ are used in the experiment. The spectrum of the broadband source used and the reflected spectrum from the undisturbed FBG sensor array are shown in Fig. 2(a) and Fig. 2(b) respectively. The peak power of the reflected signals depends on the broadband source's intensity distribution, which has a peak intensity at $1560 \mathrm{~nm}$, and the reflectivities of the FBGs. The reflectivity of the FBGs used in this 


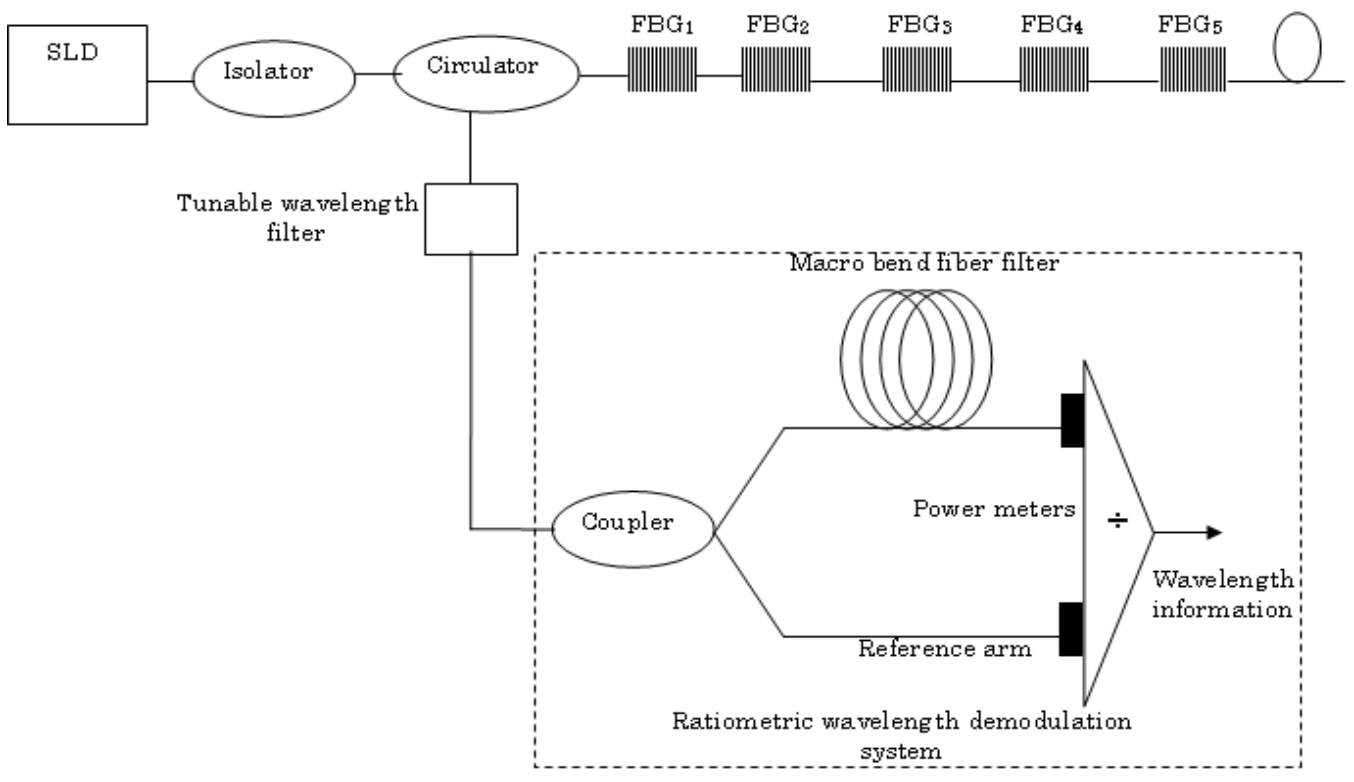

Figure 1. Schematic of the interrogations system with multiple FBGs

experiment was approximately $90 \%$. At the receiver end two identical photodiodes are used together with low noise preamplifiers, as the signals reflected from the FBGs are of a very low power and will be further attenuated by the fiber filter. The data acquisition card used to acquire data from the photodiodes was a NI6143 card which can give a sampling rate up to $250 \mathrm{kS} / \mathrm{s}$. The bandwidth of the preamplifier used was $2 \mathrm{kHz}$. The

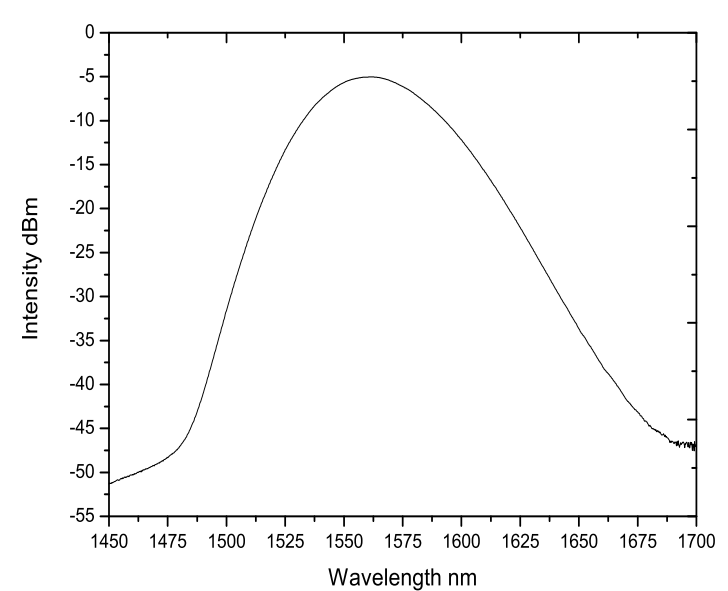

(a)

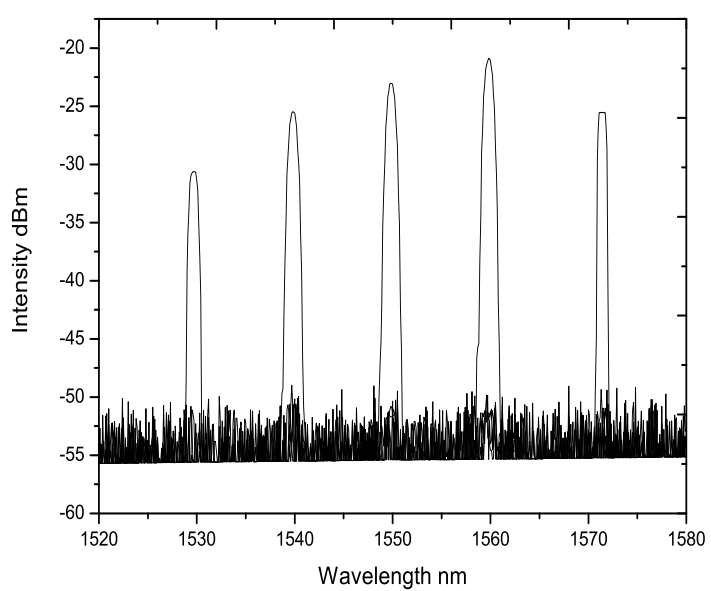

(b)

Figure 2. (a) Spectral distribution of the broadband source used (b) Reflected spectra from the FBGs 
macro-bend fiber demodulation system consists of only passive devices and therefore it does not affect the measurement speed. Thus dynamic strain can be measured up to a frequency limit imposed by the speed of the post-detection electronics. The tunable filter used in this experiment was a manual optical tunable filter whose scanning speed is not considered important in this study as the primary aim of this study is focussed on the optimization of the fiber filter for interrogating multiple FBGs.

In this experiment a macro-bend fiber filter based on SMF28 fiber is used as the edge filter. Five filters are considered with a bend radius of $10.5 \mathrm{~mm}$ and with $5,10,15$, 20 and 25 bend turns which gives an average slope of $0.07 \mathrm{~dB} / \mathrm{nm}, 0.14 \mathrm{~dB} / \mathrm{nm}, 0.21$ $\mathrm{dB} / \mathrm{nm}, 0.28 \mathrm{~dB} / \mathrm{nm}, 0.35 \mathrm{~dB} / \mathrm{nm}$ respectively. The bend radius of $10.5 \mathrm{~mm}$ is chosen because it provided a useful discrimination range over the wavelength range of interest $[12,19]$. The measured ratio response of the system with different filters is shown in Fig. 3 , which is obtained at a temperature of $20{ }^{\circ} \mathrm{C}$. The measurable wavelength range is set by the signal-to-noise ratio of the source and the slope of the fiber filter [20]. From Fig. 3 it is clear that for filters with a higher number of turns, the slope is higher. However a filter with large number of turns cannot guarantee a high resolution and accuracy as it is determined by the parameters such as polarization, temperature and noise $[13,15$, 17]. For a single FBG it is possible to optimize the edge filter slope in the vicinity of the FBG peak wavelength to achieve the best possible resolution and accuracy. However for an array of FBGs with peak wavelengths spread over a wide range it is not possible to adopt a single slope that yields the best resolution and accuracy for all the FBGs. Thus for a macro-bend fiber based demodulation system, it is important to determine a best fit slope which is most suitable for a wide wavelength range and which provides the best compromise for resolution and accuracy for all the FBGs in the array. Analysis of the

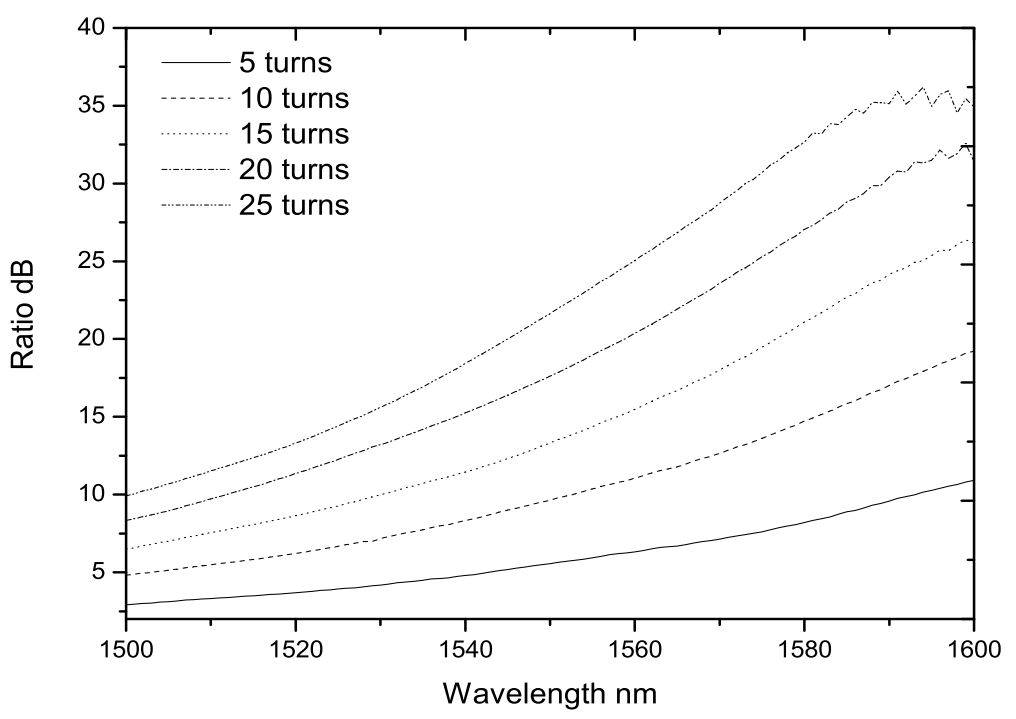

Figure 3. Ratio response of the system with different fiber filters 
system and selection of a suitable fiber filter considering all these factors are presented in the section below.

\section{Analysis of the system}

Assuming the BBS has a smooth spectral profile, its spectral response can be modelled as a Gaussian distribution of wavelengths with a spectral full-width at half-maximum of $\Delta \lambda_{0}$ and a center wavelength of $\lambda_{0}$ and can be expressed as [21],

$$
B(\lambda)=I_{\text {peak }} \exp \left[-4 \ln 2 \frac{\left(\lambda-\lambda_{0}\right)^{2}}{\Delta \lambda_{0}^{2}}\right]
$$

where $\lambda$ is the wavelength in vacuum and $I_{\text {peak }}$ is the peak power which can be expressed as,

$$
I_{\text {peak }}=\frac{P_{0}}{\Delta \lambda_{0}}[4 \ln 2 / \pi]^{1 / 2},
$$

where $P_{0}$ is the total power injected into the system by the BBS.

To simplify the calculation we have assumed that the Bragg grating reflectivity function is a Gaussian function with a center wavelength as the Bragg wavelength $\lambda_{b}$ and an FWHM of $\Delta \lambda_{b}$ and is expressed as,

$$
G_{\lambda_{b}}(\lambda)=R_{0} \exp \left[-4 \ln 2 \frac{\left(\lambda-\lambda_{b}\right)^{2}}{\Delta \lambda_{b}^{2}}\right]
$$

where $R_{0}$ is the maximum reflectivity that occurs at the Bragg wavelength.

It has to be noted that in practice the reflected signal from the FBG has a limited SNR. The limited SNR of the signal fed to the demodulation system will affect the linearity of the measured ratio spectrum and hence the wavelength range [20]. The spectral width of the BBS is much larger than the spectral width of the FBG, hence we can express the spectral distribution of the wavelengths reflected from a grating when illuminated by the BBS with a limited SNR as,

$$
I_{b}(\lambda)=\left[\begin{array}{cc}
B_{\lambda_{b}} G_{\lambda_{b}}, & \left|\lambda-\lambda_{b}\right| \leq \Omega \\
-S+\text { Rand.Rs }, & \left|\lambda-\lambda_{b}\right|>\Omega
\end{array}\right],
$$

where $B\left(\lambda_{b}\right)$ is given by equation 1 , when $\lambda=\lambda_{b}$ and $S$ is the SNR of the reflected signal from the FBG. To describe the random fluctuations in the noise floor of the reflected signal, the term Rand.Rs is used, where Rand is a random number (between +0.5 and -0.5$)$ and $R s$ is a parameter which dictates the peak fluctuation in the SNR. $\Omega$ is a parameter which is determined by the noise level and can be determined for any reflected signal with a given SNR from the relationship,

$$
\left.B_{\lambda_{b}} R_{0} \exp \left(-4 \ln 2 \frac{\Omega^{2}}{\Delta \lambda_{b}^{2}}\right)\right]=-S
$$

Receiver noise also has an impact on the ratio of the system and it is necessary to consider the noise effects while modelling the system. Taking account of noise, the total 
power reaching the photodiodes connected to the filter arm and the reference arm can be represented as follows,

$$
\begin{aligned}
& I_{f}(\lambda)=\int I_{s}(\lambda) S_{1}(\lambda) T_{f}(\lambda) d \lambda+\text { Grand }_{e} \\
& I_{r}(\lambda)=\int I_{s}(\lambda) S_{2}(\lambda) d \lambda+\text { Grand }_{r},
\end{aligned}
$$

where $S_{1}(\lambda)$ and $S_{2}(\lambda)$ represents the responses of the arms of the $3 \mathrm{~dB}$ coupler. Gaussian statistics are used to model the electrical noise. Grand and $_{\text {Grand }}$ are Gaussian random numbers used to represent the receiver noise with a mean value 0 and standard deviation equal to the rms noise of the receivers connected to the edge filter and the reference arms respectively. The noise in the two receivers employed are uncorrelated to each other. As the shot noise of the receiver connected to the edge filter is a function of optical power and thus wavelength, so Grand $_{e}$ varies as the wavelength changes [17]. Since the power distribution of the BBS source also varies with wavelength, Grand $_{e}$ and Grand $_{r}$ changes for different peak reflected wavelengths.

Considering the BBS spectral distribution, noise in the receiver and the SNR of the reflected Bragg wavelength spectrum, the ratio of the system at a wavelength, $\lambda_{0}$, can be expressed as,

$$
R\left(\lambda_{0}\right)=-10 \log _{10}\left[\frac{I_{f \lambda_{0}}}{I_{r \lambda_{0}}}\right]
$$

\subsection{Effect of noise in the system}

Using the above model, the ratio fluctuation in the system for all the 5 bend loss filters and for all the peak reflected wavelengths from the FBG, caused by the noise in the receivers and limited SNR of the FBG signal are calculated. The estimated ratio fluctuation for all the FBG sensors is shown in Fig 4(a). From the figure we can see that the ratio fluctuation is significantly higher, for example, at $1550 \mathrm{~nm}$, the ratio fluctuation is $0.03 \mathrm{~dB}$ for a filter with 10 fiber turns. Due to this high ratio fluctuation, the system cannot repeatably resolve small strain or temperature changes and hence averaging can be used to reduce the ratio fluctuation. The ratio fluctuation of the system with an averaging of 256 is shown in Fig. 4(b) which is in the acceptable range to resolve small strain and temperature changes. However, as a consequence of averaging, the measurement speed of the system will be reduced and that will affect the dynamic measurement capabilities of the system.

Due to the noise induced fluctuation in the ratio of the system, the resolution of the system will be limited and will be a function of wavelength and filter discrimination. The estimated resolution of the system, which is limited by the noise of the receiver and SNR of the reflected Bragg signal, is shown in Fig. 5(a) with an averaging over 256 samples. From Fig 5(a) it can be seen that for FBGs with peak wavelengths around $1540.15 \mathrm{~nm}, 1550.2 \mathrm{~nm}$ and $1571.4 \mathrm{~nm}$, a filter with 5 turns gives the best achievable resolution among the 5 filters, while for $1530.4 \mathrm{~nm}$ and $1560.1 \mathrm{~nm}$, a filter with 10 turns 


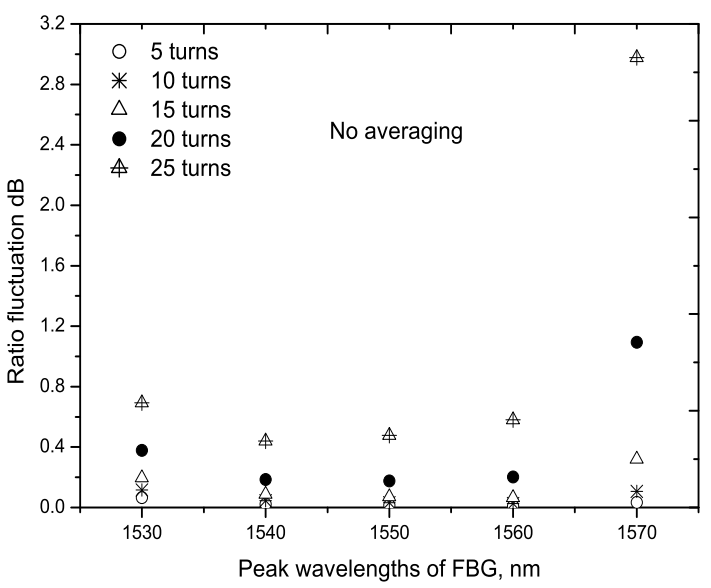

(a)

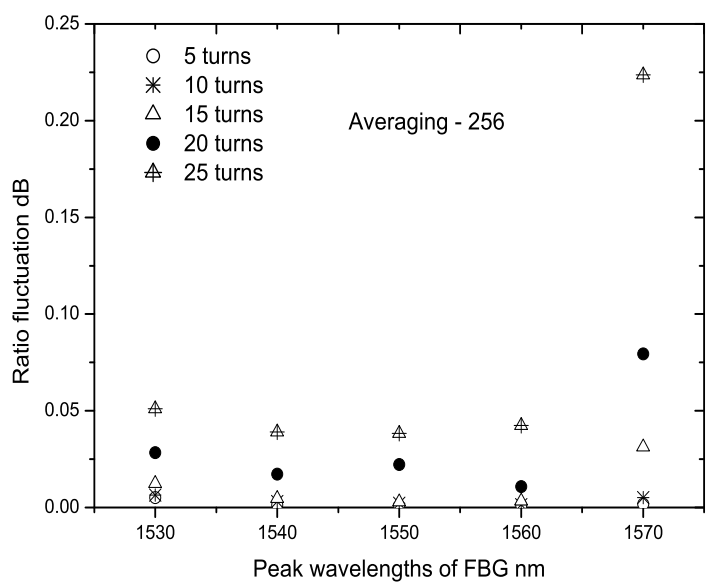

(b)

Figure 4. Fluctuation in ratio due to the noise at different FBG peak wavelengths for systems with different slopes (a) without averaging (b) with 256 averaging

gives the highest resolution. Hence the selection of a suitable filter can be made based on calculations of the deviation of the estimated resolution of each filter from the best individual resolution for each FBG. The calculated percentage deviation in resolution for systems with filters of 5 and 10 turns for all the FBG peak wavelengths from the

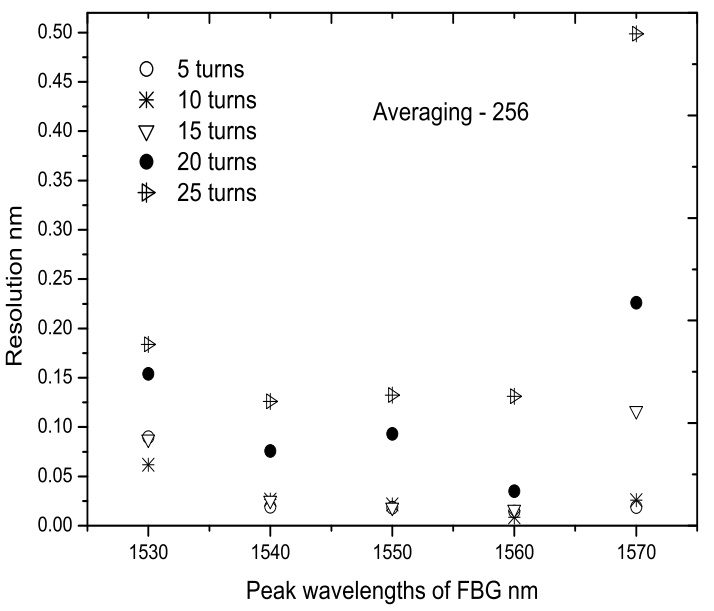

(a)

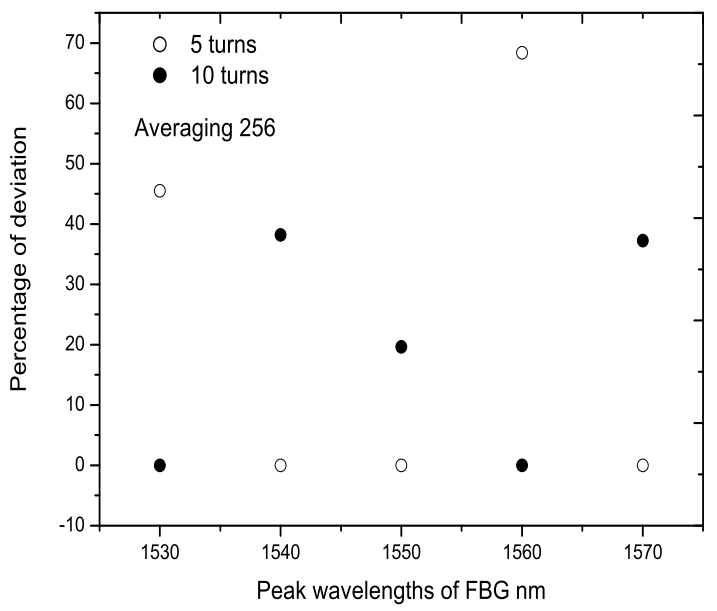

(b)

Figure 5. (a) Estimated resolution for FBGs with different peak wavelengths with different system slopes with an averaging of 256 (b) Percentage deviation from the best resolution for different FBGs for system with filters of 5 and 10 turns 


\begin{tabular}{cccc}
\hline $\begin{array}{c}\text { System } \\
\text { with different } \\
\text { filters }\end{array}$ & $\begin{array}{c}\text { Bragg } \\
\text { Wavelengths } \\
\mathrm{nm}\end{array}$ & $\begin{array}{c}\text { Strain } \\
\text { Resolution } \\
\mu \varepsilon\end{array}$ & $\begin{array}{c}\text { Temperature } \\
\text { Resolution } \\
{ }^{0} \mathrm{C}\end{array}$ \\
\hline \multirow{5}{*}{5 turns } & 1530.40 & 74.88 & 8.16 \\
& 1540.15 & 15.93 & 1.73 \\
& 1550.20 & 15.13 & 1.65 \\
& 1560.10 & 11.87 & 1.29 \\
& 1571.40 & 15.69 & 1.71 \\
& & & \\
& 1530.40 & 51.45 & 5.61 \\
& 1540.15 & 22.02 & 2.40 \\
& 1550.20 & 18.11 & 1.97 \\
& 1560.10 & 7.05 & 0.76 \\
& 1570.40 & 21.53 & 2.34 \\
\hline
\end{tabular}

Table 1. Estimated strain and temperature resolution of the system with filters of 5 and 10 fiber turns

individual best resolution is shown in Fig 5(b). From the figure it can be seen that for a filter with 5 turns, $\mathrm{FBG}_{2}, \mathrm{FBG}_{3}$ and $\mathrm{FBG}_{5}$ can have the highest resolution, while the other two have the largest deviation from the best achievable value. However for a system with filter with 10 turns $\mathrm{FBG}_{1}$ and $\mathrm{FBG}_{4}$ can have much better resolutions. Filters with 15, 20 and 25 bend turns are not considered for further analysis due to its high ratio fluctuation when compared to filters of 5 and 10 bend turns.

Ultimately the measurement system is required not to just measure wavelength but also to provide a measure of strain or temperature and for this reason it is necessary to estimate the strain and temperature resolution of the interrogation system. The strain and temperature sensitivity of the FBGs used were $1.2 p m / \mu \varepsilon$ and $11 \mathrm{pm} /{ }^{0} \mathrm{C}$ respectively. The estimated strain and temperature resolution for all 5 FBGs obtained with systems using filters with 5 and 10 turns are shown in Table 1 . The average strain and temperature resolution of the system with 5 turns is $26.7 \mu \varepsilon$ and $2.9{ }^{\circ} C$ respectively while for a system with 10 turns the resolution is $24.03 \mu \varepsilon$ and $2.61{ }^{0} C$ respectively. While these results suggest that the 10 turns filter is best, the effect of polarization and temperature drift for the system and their impact on accuracy also needs to be considered before making a final determination of which filter to use.

\subsection{Effect of polarization and temperature dependency of the system}

The polarization and temperature dependency of the macro-bend fiber are the key parameters determining the accuracy of the interrogation system. The polarization dependency of the macro-bend fiber originates from the difference in bend loss for the TE mode and TM mode propagating along the fiber [13]. This occurs because of the different boundary conditions between the cladding and polymer coating layers for the 


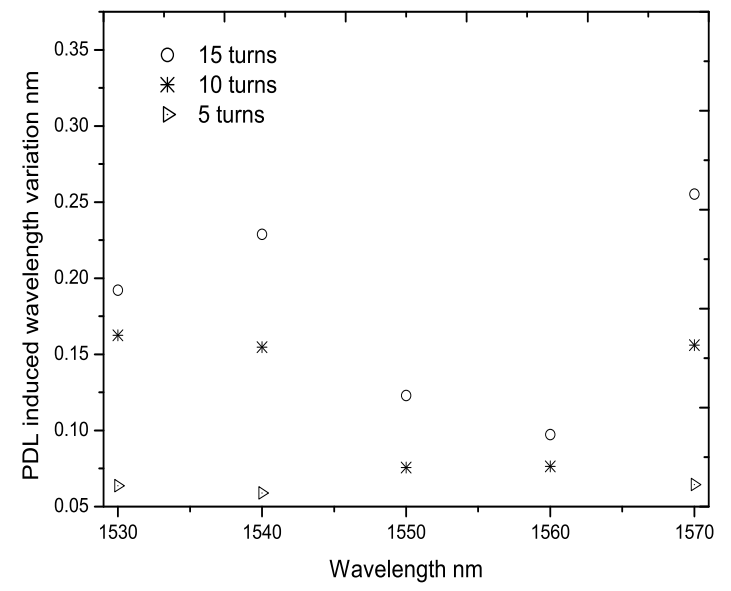

(a)

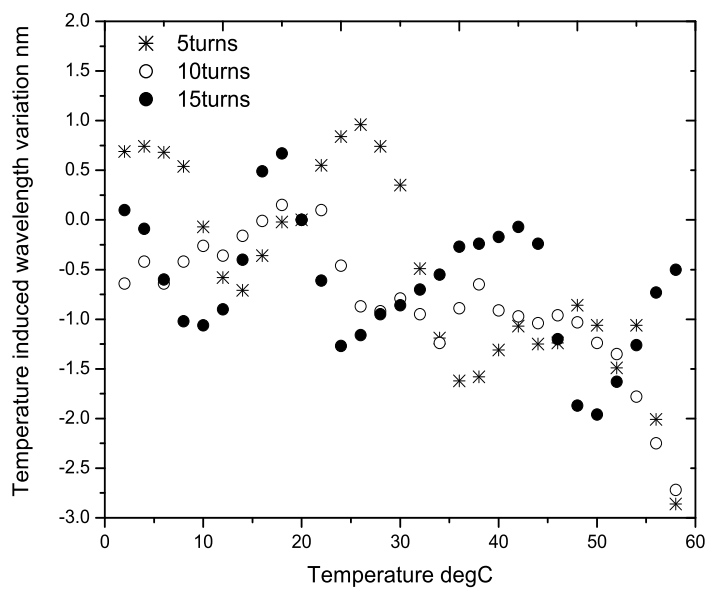

(b)

Figure 6. (a) Measured inaccuracy at the peak reflected wavelengths of the FBG due the PDL of the system (b) Temperature induced wavelength variation of the system with different filters at $1550 \mathrm{~nm}$

two polarization states [13]. However the polarization dependency of the fiber filter can be minimized by using a twisted configuration as we proposed in Ref [22]. Since the PDL of the filter originates from the difference in bend loss for the TE and TE modes, a $90^{\circ}$ twist in the middle of the fiber will effectively equalize the losses for the input TE and TM modes and the PDL of the whole section will be minimized. Commonly all commercial $3 \mathrm{~dB}$ couplers exhibit a finite PDL. To minimize the PDL of the $3 \mathrm{~dB}$ coupler, a polarization insensitive (PI) $3 \mathrm{~dB}$ coupler is used together with the twisted fiber filter in the interrogation system $[22,23]$. Even with the twisted filter configuration and the PI $3 \mathrm{~dB}$ coupler, a small finite PDL induced ratio error remains in the system due to the residual PDL of the fiber filter which originates due to the small mismatch between the two bend fiber sections and also due to the finite small PDL (0.01 - 0.02 $\mathrm{dB}$ ) of the PI $3 \mathrm{~dB}$ coupler. This residual PDL can result in wavelength inaccuracies. The measured wavelength inaccuracies due to the PDL of the system with filters of 5 , 10, 15 turns are shown in Fig. 6(a).

Ambient temperature variation is another factor that can create inaccuracies in the measured wavelength of systems based on macro-bend fiber filters. Earlier investigations on macro-bend fiber edge filters show that the buffer coating layer has a significant effect on the temperature dependence of the filter $[15,16,24]$. Due to whispering gallery modes induced oscillatory variations in the bend loss and hence in the ratio, and the different ratio variations at different wavelengths, a correction in the calibration response required to compensate for temperature variation is very complex for systems based on SMF28 fiber filter. For illustration the temperature induced wavelength variation of the system with different fiber filters at $1550 \mathrm{~nm}$ is shown in Fig. 6(b) which is oscillatory in nature. 


\begin{tabular}{cccccc}
\hline $\begin{array}{c}\text { System } \\
\text { with different } \\
\text { filters }\end{array}$ & $\begin{array}{c}\text { Bragg } \\
\text { Wavelengths }\end{array}$ & $\begin{array}{c}\text { PDL induced } \\
\text { inaccuracy }\end{array}$ & \multicolumn{3}{c}{$\begin{array}{c}\text { Temperature induced } \\
\text { inaccuracy }\end{array}$} \\
\hline & 1530.40 & \pm 53 & \pm 5.78 & \pm 5.58 & \pm 0.60 \\
5 turns & 1540.15 & \pm 49.08 & \pm 5.35 & \pm 6.83 & \pm 0.75 \\
& 1550.20 & \pm 13 & \pm 1.41 & \pm 8.23 & \pm 0.90 \\
& 1560.10 & \pm 26.16 & \pm 2.85 & \pm 20.91 & \pm 1.19 \\
& 1571.40 & \pm 53.75 & \pm 5.86 & \pm 13.25 & \pm 1.45 \\
& & & & & \\
& 1530.40 & \pm 135.41 & \pm 14.77 & \pm 6.75 & \pm 0.73 \\
& 1540.15 & \pm 129 & \pm 14.07 & \pm 6.57 & \pm 0.72 \\
& 1550.20 & \pm 63 & \pm 6.87 & \pm 9.34 & \pm 1.01 \\
& 1560.10 & \pm 63.66 & \pm 6.94 & \pm 9.25 & \pm 1.01 \\
& 1570.40 & \pm 130.08 & \pm 14.19 & \pm 12.41 & \pm 1.35 \\
\hline
\end{tabular}

Table 2. Estimated accuracy of the system with filters of 5 and 10 turns due to the polarization and temperature dependence of the system

Therefore for the interrogation system to achieve the highest accuracy, the temperature of the fiber filter has to be maintained at a temperature equal to that used during calibration.

The wavelength error due to the polarization and temperature dependence of the system leads to measurement inaccuracies in strain and temperature. The inaccuracies in strain and temperature measurements due to the PDL of the system and a temperature variation $\pm 1{ }^{0} \mathrm{C}$ from the calibration temperature of $20{ }^{0} \mathrm{C}$ for fiber filters of 5 and 10 turns are estimated. Table 2 summarize the estimated accuracy of strain and temperature measurements for all the 5 FBGs when used with the demodulation system with filters of 5 and 10 turns. From table 1 and table 2 one can see that the average strain and temperature resolution of a system with 10 turns is slightly higher when compared to 5 turns, but the accuracy is very poor for a system with 10 turns compared to 5 turns. The average strain inaccuracy induced by PDL and temperature dependence for a system with 5 turns are $\pm 38.23 \mu \varepsilon$ and $\pm 8.96 \mu \varepsilon$ respectively while for a system with 10 turns the accuracies are $\pm 104.23 \mu \varepsilon$ and $\pm 8.87 \mu \varepsilon$ respectively. Similarly the average accuracy in measured temperature induced by the polarization and temperature dependence of the system with 5 fiber turns is $\pm 4.25{ }^{\circ} \mathrm{C}$ and $\pm 0.97{ }^{0} \mathrm{C}$ respectively, while a system with 10 turns is $\pm 11.37{ }^{\circ} \mathrm{C}$ and $\pm 0.96{ }^{\circ} \mathrm{Crespectively.} \mathrm{Thus}$ from the analysis we conclude that while the average resolution is marginally higher for the 10 turn filter, the accuracy of the system taking into account PDL is substantially better for a filter with 5 turns and for this reason this filter is selected as the one which can give the overall best results for the FBG array. An experimental performance evaluation of the interrogation system with a fiber filter of 5 turns is presented in the next section. 


\section{Performance evaluation of the system}

For evaluating the performance of the interrogation system, which is estimated in the above section, static strain, dynamic strain and temperature are measured using the FBG interrogation system. In our experiment, temperature variations are applied to $F B G_{2}$, static strain to $F B G_{3}$ and dynamic strain to $F B G_{4}$ whose reflected wavelengths are $1540.15 \mathrm{~nm}, 1550.2 \mathrm{~nm}$ and $1560.1 \mathrm{~nm}$ respectively. The other 2 FBGs are left undisturbed for the purpose of this experiment. The fiber filter used in the system has 5 fiber turns. For all the measurements averaging over 256 samples was applied. The temperature of the fiber filter is monitored during the course of the experiment and the recorded variation in the ambient temperature was $\pm 2{ }^{\circ} \mathrm{C}$ around $20{ }^{\circ} \mathrm{C}$.

\subsection{Static and dynamic strain measurements}

For applying static strain to $F B G_{3}$, one end of the grating is fixed to a translation stage and other end to a fixed point. Using a micrometer translation stage a strain up to $540 \mu \epsilon$ is applied to the FBG using incremental steps of $90 \mu \epsilon$. The applied strain is calculated from the ratio of the elongation of the fiber containing the FBG, which is the same as the micrometer translation value and from the length of the fiber between the fixed points. This applied strain is measured with the macro-bend fiber based interrogation system. For strain measurements the temperature of the FBG is kept constant using a temperature controller, which has an accuracy of $\pm 0.1{ }^{0} C$. This temperature variation can contribute an inaccuracy of $\pm 1 \mu \varepsilon$ to the strain measurements. Fig. 7 shows the comparison between the measured and applied static strain. Both are in close agreement, demonstrating that the system measures strain accurately. To examine the resolution of the system, step strains of $10 \mu \epsilon$ and $20 \mu \epsilon$ are applied to the FBG. The measured

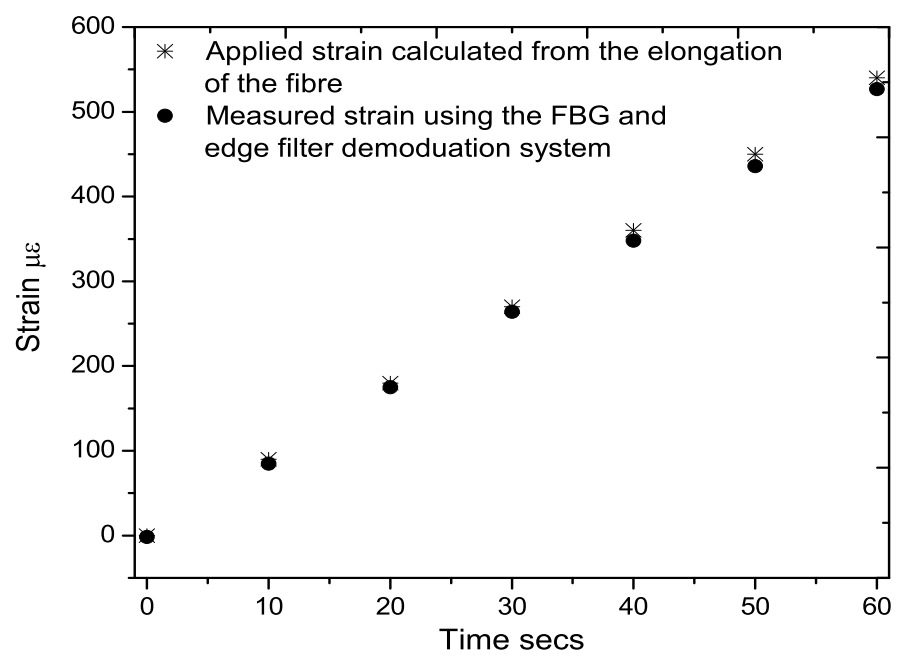

Figure 7. A comparison between measured and applied strain 


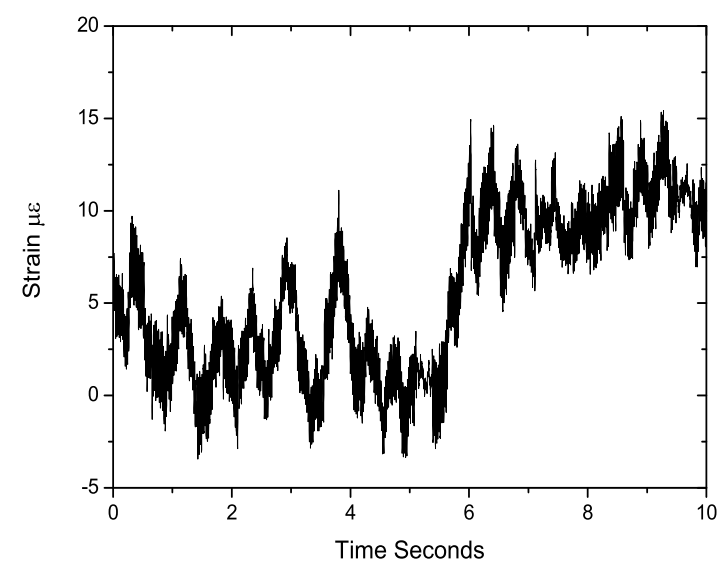

(a)

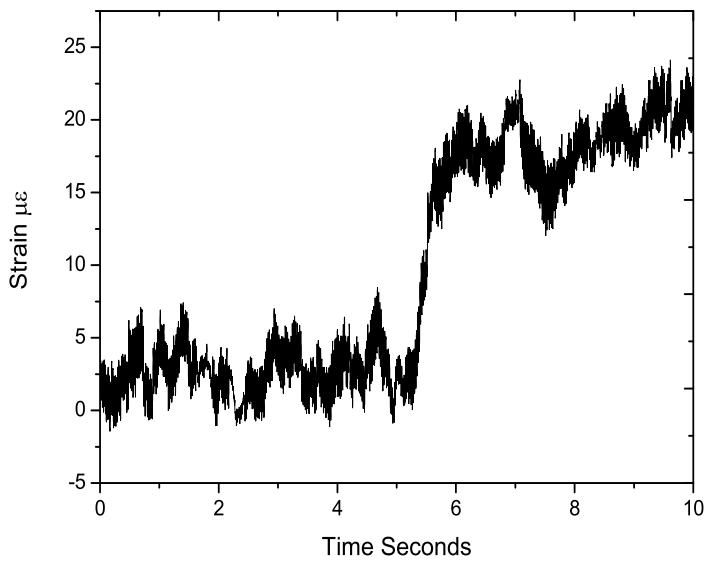

(b)

Figure 8. Measured static strain for a step change of (a) $10 \mu \varepsilon$ (b)20 $\mu \varepsilon$

strain variations are shown in Fig. 8(a) and Fig. 8(b) respectively. From the figure it can be seen that $20 \mu \epsilon$ is clearly detectable and hence the measured strain resolution agrees with the estimated strain resolution of $15.13 \mu \epsilon$ for a system with a filter of 5 fiber turns.

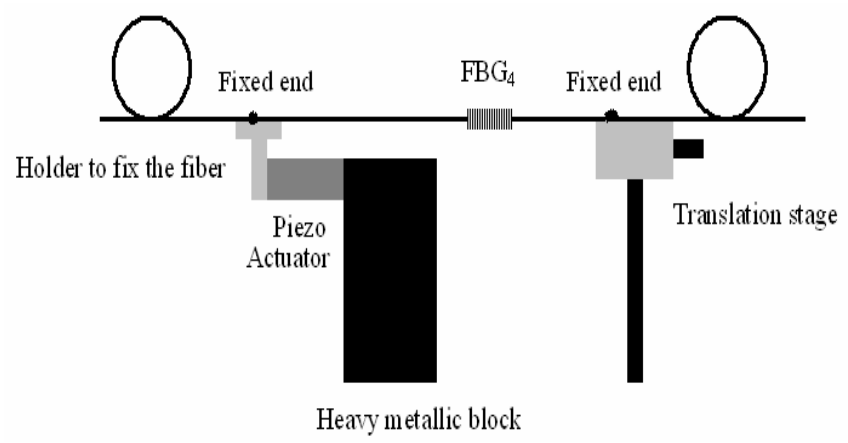

Figure 9. Schematic of the setup to apply dynamic strain to the FBG.

To apply dynamic strain, as shown in Fig. 9, a piezo actuator (AE0505D18) is used, where one surface of the piezo actuator is glued to a heavy fixed surface. The other end of the piezo actuator is fixed to a $\mathrm{T}$ shaped element to which one end of the fiber containing the FBG is attached. The other end of the fiber is attached to a micrometer translation stage, which is used to adjust the static pre-strain. The driver used to supply voltage to the piezo actuator was a MDT694A from Thorlabs. The output current versus frequency characteristic of the driver also needs to be considered for experimental measurements. For the driver used the maximum output current was $60 \mathrm{~mA}$. The maximum sinusoidal frequency that can give the same displacement for a 


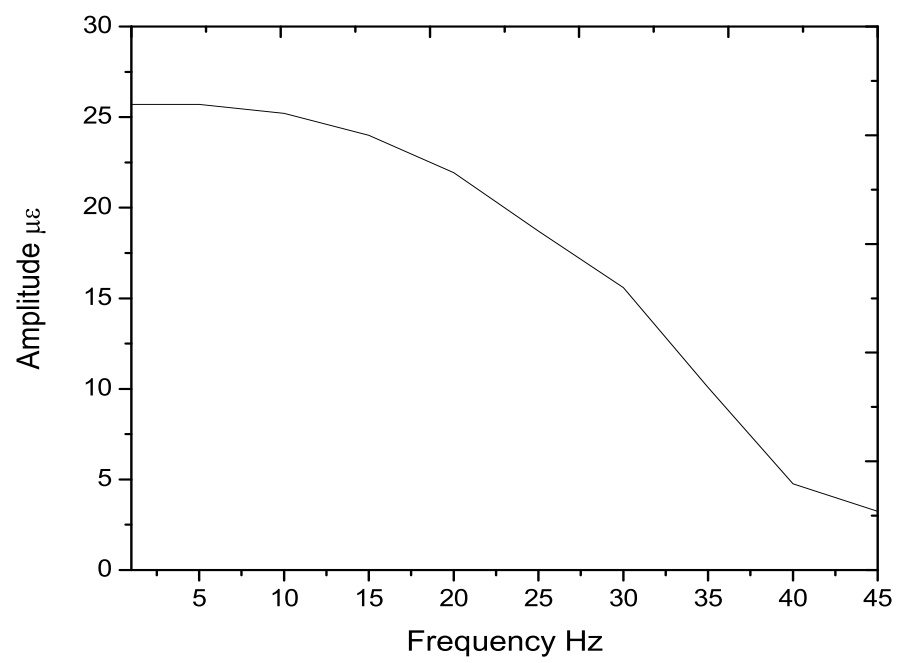

Figure 10. Strain amplitude versus frequency characteristics of the piezo driver for a $0 \mathrm{~Hz}$ strain of $25 \mu \epsilon$.

current $I_{\text {out }}$ can be calculated from the relation, $f=\frac{I_{\text {out }}}{2 \pi V_{\text {amp }} C}$, where $V_{\text {amp }}$ is the voltage applied to the piezo actuator and $\mathrm{C}$ is the capacitance of the piezo actuator. The strain amplitude versus frequency characteristic for the driver is shown in Fig. 10, for a $0 \mathrm{~Hz}$ (or DC) strain value of $25 \mu \epsilon$.

Different amplitudes of dynamic strain at a frequency $25 \mathrm{~Hz}$ were applied to $F B G_{4}$. Fig. 11 shows the measured dynamic strain with an applied strain amplitude of $19 \mu \epsilon$ (equivalent to $25 \mu \epsilon$ at $0 \mathrm{~Hz}$ ) with a variation of $\pm 7 \mu \epsilon$. The dynamic strain resolution

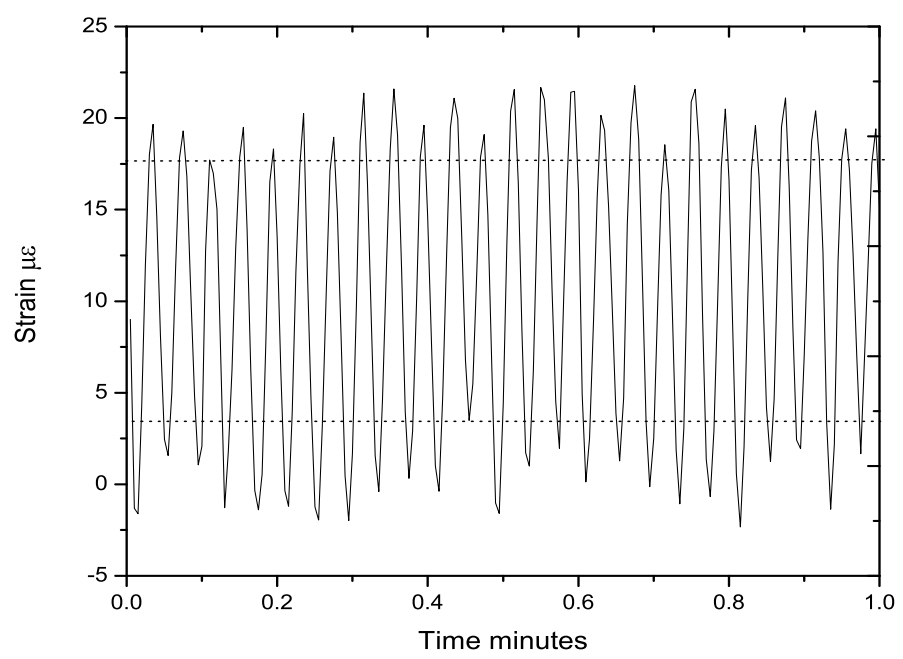

Figure 11. Measured dynamic strain for a strain amplitude of $19 \mu \epsilon$ and frequency $25 \mathrm{~Hz}$ 
measured was $5 \mu \epsilon / \sqrt{H} z$. Applying dynamic strain at higher frequencies was not possible due to the limitations of the piezo driver to provide the required current to drive the piezo actuator at higher frequencies. In summary, using a FBG sensor array together with a fiber bend loss filter demodulation system, static and dynamic strain measurement is clearly achievable.

\subsection{Temperature measurements}

To demonstrate the ability of the interrogation system to measure the temperature variations, $F B G_{2}$ with a peak reflected wavelength of $1540.15 \mathrm{~nm}$ was subjected to temperature variations by attaching the sensor to a Peltier cooler which was driven by a temperature controller. To verify the temperature resolution of the system, incremental step changes of $2{ }^{0} \mathrm{C}$ have been applied from $20{ }^{0} \mathrm{C}$ to $30{ }^{0} \mathrm{C}$. As the temperature controller requires time to settle, the measurements were taken at 2 minute time intervals. The measured change in temperature and a comparison with the temperature measured by the temperature controller are shown in Fig. 12. The comparison shows that a temperature resolution of $2{ }^{0} \mathrm{C}$ can be obtained with the interrogation system which was predicted for a system with an FBG sensor of peak reflected wavelength $1540.15 \mathrm{~nm}$ and with a macro-bend fiber filter of 5 turns.

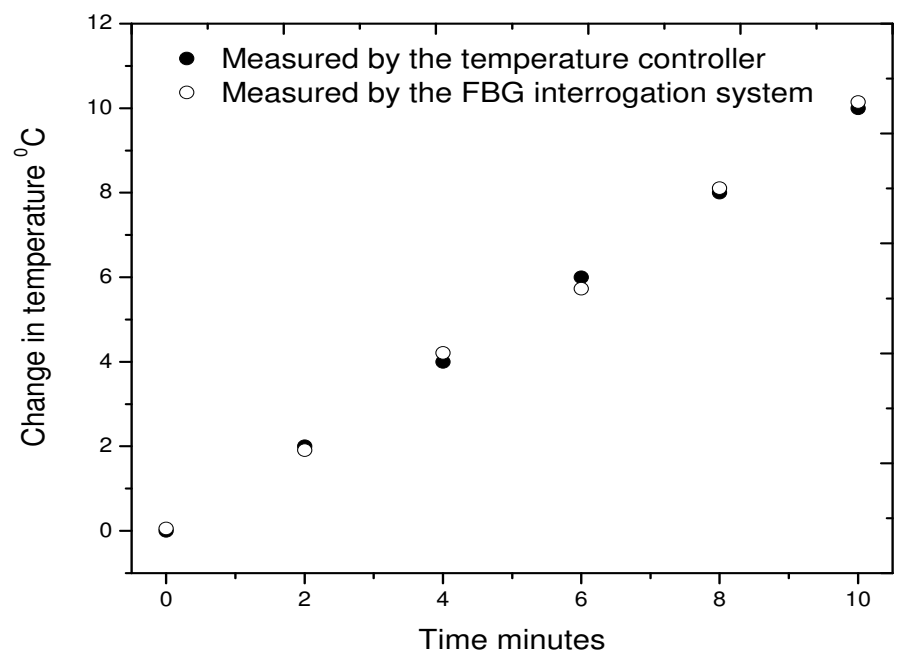

Figure 12. A comparison of change in temperature measured using the FBG interrogation system and the temperature controller

\section{Conclusion}

An analysis and performance evaluation of an all-fiber interrogation system for an FBG sensor array is presented. Due to the characteristic properties of the macro-bend fiber filter and the total noise associated with the system, it is shown that it is not possible 
to achieve the optimum strain and temperature resolution for all the FBGs in an array using a single filter with a fixed slope. The polarization and temperature dependent losses are different for different wavelengths and hence the accuracy of the measurands will be different for different FBGs. Since noise associated with the system determines the resolution of the system, which is a function of wavelength, the resolution of the measurands is different for different FBG sensors. To demonstrate these effects, we had considered a system with different filters, which give different ratio slopes. The maximum achievable resolution and accuracy for FBGs of peak reflected wavelengths at $1530.40 \mathrm{~nm}, 1540.15 \mathrm{~nm}, 1550.2 \mathrm{~nm}, 1560.1 \mathrm{~nm}, 1571.4 \mathrm{~nm}$ were estimated. From the estimation we have found that a system with a filter of 5 number of turns provides the best fit slope with which the best results for the FBG array can be achieved. Performance evaluation of the system with a macro-bend fiber filter has been carried out and the experimental results agreed with the estimated values. Temperature, static strain and dynamic strain were applied to 3 FBGs with peak wavelengths $1540.15 \mathrm{~nm}$, $1550.2 \mathrm{~nm}$ and $1560.1 \mathrm{~nm}$ respectively. In the demonstration we achieved a static strain resolution of $20 \mu \varepsilon$ and dynamic strain resolution of $5 \mu \epsilon / \sqrt{H} z$. Thus in this paper we have successfully demonstrated the consequences of the characteristic properties of the system and how it affects the resolution and accuracy of the measurands. We have also proved the importance of a best fit slope to achieve the best results for an array of FBGs, when used together with a macro-bend fiber filter demodulation system.

\section{References}

[1] Byoungho Lee 2003 Review of present status of optical fiber sensors Optical fiber technology $957-79$

[2] K. O. Hill, G Meltz 1997 Fiber Bragg grating technology fundamentals and overview J. Lightwave Technol 15 No.8

[3] R. M. Measures, S. Melle, K. Lie 1992 Wavelength demodulated Bragg grating fiber optic sensing systems for addressing smart structures critical issues Smart. Mater. Struct. 1 36-44

[4] H. J. Bong, H. K. Kang, C. S. Hong and C. G. Kim 2005 Optical fiber sensor systems for simultaneous monitoring of strain and fractures in composites Smart. Mater. Struct. 14 N52N58

[5] K. Pran, G. B. Havsgard, G. Sagvolden, O. Farsund and G. Wang 2002 Wavelength multiplexed fiber Bragg grating system for high strain health monitoring applications Meas. Sci. Technol. 13 471-476

[6] Y. Qiu, Y. Sheng, C. Beaulieu 1999 Optimal Phase Mask for Fiber Bragg Grating Fabrications $J$. Lightwave Technol 17 2366-2370

[7] S. M. Melle, K. Liu and R. M. Measures 1992 A passive wavelength demodulation system for guided Bragg grating sensors IEEE Photon. Technol. Lett. 4 516-518

[8] M. A. Davis and A. D. Kersey 1994 All fiber Bragg grating sensor demodulation technique using a wavelength division coupler Electron. Lett. 30 75-77

[9] A. B. L Ribeiro, L. A. Ferreira, M. Tsvekov and J. L Santos 1996 All-fiber interrogation technique for fiber Bragg sensors using biconical fiber filter Electron. Lett. 32 382-383

[10] Y. Liu, L. Zhang and I. Bennion 1999 Fabricating fibre edge filters with arbitrary spectral response based on tilted chirped grating structures Meas.Sci. Technol. 10 L1-L3 
[11] Y. Zhao and Y. Liao 2005 Discrimination methods and demodulation techniques for fiber Bragg grating sensors Optics and Lasers In Engineering 41 1-18

[12] Q. Wang, G. Farrell, T. Freir, G. Rajan and P. Wang 2006 Low-cost wavelength measurement based on a macrobending single-mode fiber Opt.Lett. 31 1785-1787

[13] Q. Wang, G. Rajan, P. wang, G. Farrell 2007 Polarization dependence of bend loss in a standard singlemode fiber Optics Express 15 4909-4920

[14] Rajan. G, Wang. Q, Semenova. Y, Farrell. G, Wang. P 2008 Effect of polarization dependent loss on the performance accuracy of a ratiometric wavelength measurement system IET Optoelectronics $263-68$

[15] R. Morgan, J. S. Barton, P. G. Harper, J. D. C. Jones 1990 Temperature dependece of bending loss in monomode optical fibers Electronics Letters 26 937-939

[16] P. Wang, Y. Semenova, G. Farrell 2008 Temperature dependence of macrobending loss in all-fiber bend loss edge filter Optics Communications $2824312-4316$

[17] G. Rajan, Y. Semenova, T. Freir, P. Wang, G. Farrell 2008 Modelling and analysis of the effect of noise on an edge filter based ratiometric wavelength measurement system J. Lightwave Technol in press, accepted for publication

[18] Y. J. Rao 1997 In-fibre Bragg grating sensors Meas, Sci.Technol. 8 355-375

[19] Q. Wang, G. Farrell and T. Freir 2005 Theoretical and experimental investigations of macro-bend losses for standard single mode fibers Optics Express 13 4476-4484

[20] Q. Wang, G. Farrell and T. Frier 2005 Study of transmission response of edge filters employed in wavelength measurements Applied Optics 44 7789-7792

[21] A. B. L. Ribeiro, L. A Ferreira, L. Santos, D. A Jackson 1997 Analysis of the reflective-matched fiber Bragg grating sensing interrogation scheme Applied Optics 36 934-939

[22] G. Rajan, Y. Semenova, G. Farrell, Q. Wang, P Wang 2008 A Low polarization sensitivity all-fiber wavelength measurement system IEEE Photonics Technology Letters 20 1464-1466

[23] K. A. Murphy, M. F. Gunther, A. M. Vengsarkar 1991 Variable-ratio polarization insensitive 3x3 fused biconical tapered couplers Electronics Letters 27 1336-1337

[24] F. M. Haran, J. S Barton, S. R. Kidd, J. D. C Jones 1991 Optical fibre interferometric sensors using buffer guided light Measurement Science and Technology 5 526-530 